\title{
STELLAR COMPANIONS TO THE EXOPLANET HOST STARS HD 2638 AND HD 164509
}

\author{
Justin M. Wittrock ${ }^{1}$, Stephen R. Kane ${ }^{1}$, Elliott P. Horch $^{2}$, Lea $\mathrm{Hirsch}^{3}$, Steve B. Howell ${ }^{4}$, David R. Ciardi ${ }^{5}$, \\ Mark E. Everett ${ }^{6}$, and Johanna K. Teske ${ }^{7}$ \\ ${ }^{1}$ Department of Physics \& Astronomy, San Francisco State University, 1600 Holloway Avenue, San Francisco, CA 94132, USA; jwittroc@mail.sfsu.edu \\ ${ }^{2}$ Department of Physics, Southern Connecticut State University, New Haven, CT 06515, USA \\ 3 Astronomy Department, University of California at Berkeley, Berkeley, CA 94720, USA \\ ${ }^{4}$ NASA Ames Research Center, Moffett Field, CA 94035, USA \\ 5 NASA Exoplanet Science Institute, Caltech, MS 100-22, 770 South Wilson Avenue, Pasadena, CA 91125, USA \\ ${ }^{6}$ National Optical Astronomy Observatory, 950 N. Cherry Avenue, Tucson, AZ 85719, USA \\ ${ }^{7}$ Carnegie Department of Terrestrial Magnetism, 5241 Broad Branch Road, NW, Washington, DC 20015, USA \\ Received 2016 June 23; revised 2016 August 29; accepted 2016 August 30; published 2016 October 31
}

\begin{abstract}
An important aspect of searching for exoplanets is understanding the binarity of the host stars. It is particularly important, because nearly half of the solar-like stars within our own Milky Way are part of binary or multiple systems. Moreover, the presence of two or more stars within a system can place further constraints on planetary formation, evolution, and orbital dynamics. As part of our survey of almost a hundred host stars, we obtained images at 692 and $880 \mathrm{~nm}$ bands using the Differential Speckle Survey Instrument (DSSI) at the Gemini-North Observatory. From our survey, we detect stellar companions to HD 2638 and HD 164509. The stellar companion to HD 2638 has been previously detected, but the companion to HD 164509 is a newly discovered companion. The angular separation for HD 2638 is $0.512 \pm 0$ ". 002 and for HD 164509 is $0.697 \pm 0$ " 002 . This corresponds to a projected separation of $25.6 \pm 1.9$ au and $36.5 \pm 1.9$ au, respectively. By employing stellar isochrone models, we estimate the mass of the stellar companions of HD 2638 and HD 164509 to be $0.483 \pm 0.007 M_{\odot}$ and $0.416 \pm 0.007 M_{\odot}$, respectively, and their effective temperatures to be $3570 \pm 8 \mathrm{~K}$ and $3450 \pm 7 \mathrm{~K}$, respectively. These results are consistent with the detected companions being late-type M dwarfs.
\end{abstract}

Key words: planetary systems - stars: individual (HD 2638, HD 164509) - techniques: high angular resolution

\section{INTRODUCTION}

Much of the focus in the exoplanetary field still lies in the detection of planets using a variety of techniques, such as radial velocity (RV) signatures, transits, direct imaging, and microlensing, among others. A significant factor that can affect the detection of exoplanets is the binarity of the host stars. In fact, it is believed that nearly half of all Sun-like stars are part of a multiple-star system (Raghavan et al. 2010). This high-rate of multiplicity has also been found in exoplanet host stars through follow-up of Kepler candidates (Everett et al. 2015; Kraus et al. 2016) and Robo-AO observations of RV exoplanet host stars (Riddle et al. 2015).

The mere presence of a binary companion can substantially affect astrometric and RV measurements of the host star, and cause severe blended contamination for transit experiments (Cartier et al. 2015; Ciardi et al. 2015; Gilliland et al. 2015). It is therefore imperative to verify the multiplicity of exoplanet host stars to ensure correct interpretation of exoplanet signals. Moreover, the binarity of the stars can place further constraints on planetary formation. Holman \& Wiegert (1999) explored the orbital stability of the planets in the presence of a binary star system. Additionally, correlations between the planets' mass and their period (Zucker \& Mazeh 2002) and eccentricities (Eggenberger et al. 2004) were examined. Several binary systems have been studied, such as $\alpha$ Centauri (Benest 1988), Sirius (Benest 1989), $\eta$ Coronae Borealis (Benest 1996), and 30 Arietis B (Kane et al. 2015; Roberts et al. 2015), which provide us with rich information on orbital dynamics in an $N$-body system.

This paper presents new results on stellar companions to the exoplanet host stars HD 2638 and HD 164509. The stellar companion to HD 2638 has been previously detected and characterized (Riddle et al. 2015; Roberts et al. 2015). However, this is an independent detection, and this paper shall present an independent analysis of that system. Meanwhile, the companion to HD 164509 has not been previously reported. In Section 2 we briefly describe the properties of HD 2638 and HD 164509, along with their known exoplanets. Section 3 discusses the method of detection, the range of targets that were selected for analysis, and the details of the data reduction. Section 4 presents the results from the data analysis and stellar isochrone fitting. Section 5 explains the potential implication of those findings for the planetary systems, including limits to the eccentricities of the binary companion that allow orbital stability. Section 6 provides discussion of further work and concluding remarks.

\section{PROPERTIES OF THE HD 2638 AND HD 164509 SYSTEMS}

The detailed stellar and planetary parameters of the HD 2638 and HD 164509 systems are shown in Table 1. HD 2638 is a G5V star that is about $50 \mathrm{pc}$ away toward the constellation of Cetus (ESA 1997; van Leeuwen 2007). It is believed to be part of a wide binary system with the nearby star HD 2567. Shaya \& Olling (2011) performed a Bayesian analysis of both stars' astrometry; the result yielded a 99\% chance of both stars being true companions. However, Roberts et al. (2015) argued that, barring any errors in the measurement of the stars' parallax, they are separated by $6.8 \mathrm{pc}$, making them not gravitationally bound. HD 2638 is known to host one planet, HD 2638b, with a mass of approximately $0.48 M_{J}$ (Moutou et al. 2005). Riddle et al. (2015) discovered that HD 2638 has a stellar companion while examining the system with ROBO-AO. Roberts et al. (2015) analyzed the orbital dynamics of the primary star and 
Table 1

Stellar and Planetary Properties

\begin{tabular}{|c|c|c|}
\hline Properties & HD $2638^{a, b}$ & HD $164509^{c}$ \\
\hline \multicolumn{3}{|l|}{ Stellar } \\
\hline Spectral Type ${ }^{\mathrm{d}}$ & G5V & G5V \\
\hline$M_{\star}\left(M_{\odot}\right)^{\mathrm{e}}$ & $0.87 \pm 0.03$ & $1.10 \pm 0.01$ \\
\hline$R_{\star}\left(R_{\odot}\right)^{\mathrm{e}}$ & $0.81 \pm 0.02$ & $1.11 \pm 0.02$ \\
\hline$L_{\star}\left(L_{\odot}\right)^{\mathrm{e}}$ & $0.42 \pm 0.01$ & $1.31 \pm 0.02$ \\
\hline$T_{e}(\mathrm{~K})^{\mathrm{e}}$ & $5173 \pm 26$ & $5860 \pm 31$ \\
\hline $\log g\left(\mathrm{~cm} \mathrm{~s}^{-2}\right)^{\mathrm{e}}$ & $4.55 \pm 0.03$ & $4.38 \pm 0.02$ \\
\hline Age $(\mathrm{Gyr})^{\mathrm{e}}$ & $5.1 \pm 4.1$ & $3.2 \pm 0.8$ \\
\hline$[\mathrm{Fe} / \mathrm{H}]$ & $0.16 \pm 0.05$ & $0.21 \pm 0.03$ \\
\hline Apparent Magnitude $m_{V}^{\text {f }}$ & 9.58 & 8.24 \\
\hline 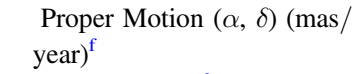 & $-105.63,-223.46$ & $-7.40,-20.98$ \\
\hline Parallax (mas) $)^{\mathrm{f}}$ & $20.03 \pm 1.49$ & $19.07 \pm 0.97$ \\
\hline Distance $(p c)^{f}$ & $49.93 \pm 3.71$ & $52.44 \pm 2.67$ \\
\hline \multicolumn{3}{|l|}{ Planetary } \\
\hline$M_{p} \sin i\left(M_{J}\right)$ & 0.48 & $0.48 \pm 0.09$ \\
\hline$P$ (Days) & $3.43752 \pm 0.00823876$ & $282.4 \pm 3.8$ \\
\hline$a(\mathrm{au})$ & 0.044 & $0.875 \pm 0.008$ \\
\hline
\end{tabular}

Notes.

${ }^{\mathrm{a}}$ Wang \& Ford (2011).

b Moutou et al. (2005).

${ }^{\mathrm{c}}$ Giguere et al. (2012).

d ESA (1997).

e Bonfanti et al. (2016)

${ }^{f}$ van Leeuwen (2007).

the stellar companion and determined that the masses of the components are $0.87 M_{\odot}$ and $0.46 M_{\odot}$, respectively. Moreover, they inferred that the spectral types are G8V and M1V and that they are separated by about $28.5 \mathrm{au}$, giving them an orbital period of around 130 years (Roberts et al. 2015). Ginski et al. (2016) performed additional astrometric and photometric analysis on the system and found that the mass of the companion star is $0.425_{-0.095}^{+0.067} M_{\odot}$.

HD 164509 is a G5V star that is about 52 pc away toward the constellation of Ophiuchus (ESA 1997; van Leeuwen 2007). It is known to host one planet, HD $164509 \mathrm{~b}$, with a mass of approximately $0.48 M_{J}$ (Giguere et al. 2012). Giguere et al. (2012, pp. 1), upon examining the RV data, found that it displays "a residual linear trend of $-5.1 \pm 0.7 \mathrm{~m} \mathrm{~s}^{-1} \mathrm{yr}^{-1}$, indicating the presence of an additional longer period companion in the system." Sirothia et al. (2014) studied this system and reported a $150 \mathrm{MHz}$ radio signature of $18 \pm 6 \mathrm{mJy}$. The authors speculated that it could be the cause of a massive moon "orbiting a rapidly rotating giant planet;" however, they emphasized that more analysis is needed before such a conclusion can be reached.

\section{OBSERVATIONS AND DATA REDUCTION}

Speckle observations of our target stars were obtained with the Differential Speckle Survey Instrument, or DSSI (Horch et al. 2009). This instrument was built at Southern Connecticut State University by one of us (E. H.), and currently enjoys official visitor instrument status at the Gemini-North Observatory. The observations were carried out as part of a larger survey program that aims to detect low-mass stellar companions to exoplanet host stars. Observations were carried out in 2014 July when $\sim 60$ targets were observed. Each measurement was acquired using two different passbands, one at $692 \mathrm{~nm}$ and another at $880 \mathrm{~nm}$. The $692 \mathrm{~nm}$ filter has an FWHM of $40 \mathrm{~nm}$, and the $880 \mathrm{~nm}$ filter has an FWHM of $50 \mathrm{~nm}$. After all images underwent data reduction, they were directly examined using the ds9 program for any bright source appearing next to the target. The two particular targets described here, HD 164509 and HD 2638, were observed during the nights of 2014 July 22 and 23 , respectively. The results from the remainder for the survey targets will be published elsewhere.

Final reconstructed images were produced from the speckle data sequences using methods that have been described in previous papers (e.g., Horch et al. 2012, 2015), but we will briefly describe the main points here. The raw speckle data are stored as FITS data cubes consisting of 1000 frames, where each frame is a $256 \times 256$ pixel image centered on the target. Frames are bias-subtracted, and then an autocorrelation is formed. These are then summed to generate a final autocorrelation for the entire observation. We Fourier transform this to obtain the spatial frequency power spectrum of the observation. The same operations are then performed on an unresolved star (effectively a point source) that lies close on the sky to the science target. By dividing the power spectrum of the science target by that of the point source, we deconvolve the effects of the speckle statistics, and arrive at a diffraction-limited estimate of the true power spectrum of the object.

Returning to the raw data frames, we next form the image bispectrum of each frame, which is the Fourier transform of the triple correlation, as described in Lohmann et al. (1983). This data product is known to contain information that can be used to calculate the phase of the object's Fourier transform, which we do using the relaxation algorithm of Meng et al. (1990). By taking the square root of the deconvolved power spectrum and combining it with this phase estimate, we generate a diffraction-limited estimate of the (complex) Fourier transform of the object. Finally, we multiply this with a Gaussian lowpass filter of width similar to the diffraction limit of the telescope, and inverse-transform to arrive at the final reconstructed image.

Using the reconstructed images, we can study the statistics of local maxima that occur as a function of separation from the central star in order to derive a detection limit curve versus separation. We follow the method described in Horch et al. (2011). By computing the average and standard deviation of the maxima inside annuli that have different mean separations from the primary star, we estimate the $5 \sigma$ detection limit as the mean value plus five times the standard deviation, converted to a magnitude difference. For Gemini data, this is done by centering annuli at distances of $0.1,0.2$, $0.3, \ldots, 1.2$ arcsec. We then use a cubic spline interpolation to develop a smooth detection limit curve at all separations in between the two extreme limits. Curves like these are shown in Figures 1 and 2.

\section{RESULTS}

Target stars that were imaged using DSSI have been examined; however, only the images of two stars, HD 2638 and HD 164509, show a nearby bright source, as can be seen in both Figures 1 and 2. Results of the DSSI observations for both stars are tabulated in Table 2. As described by Horch et al. (2015), typical uncertainties in separation for DSSI at Gemini are 1-2 mas. For a particularly faint component, such as the 

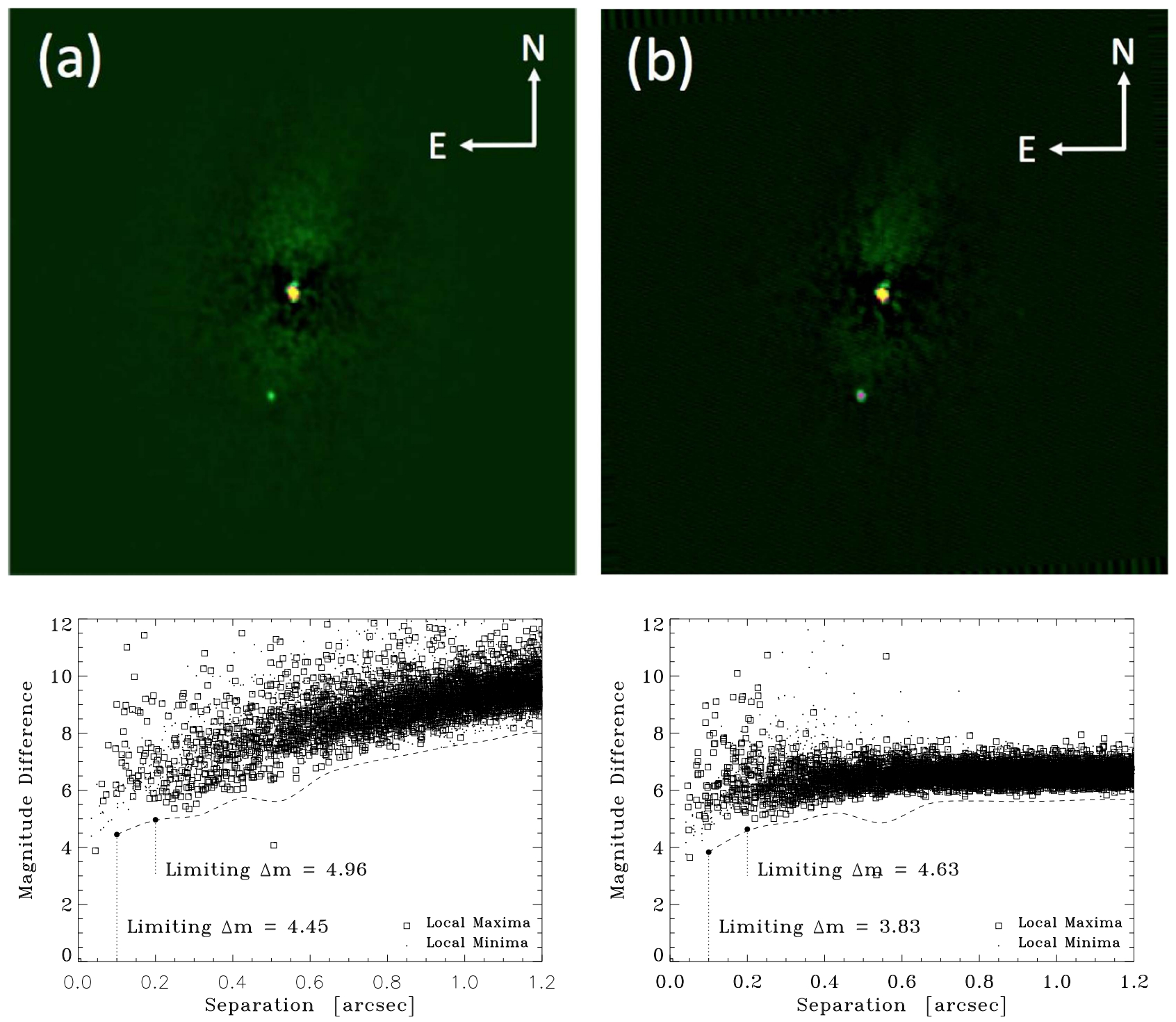

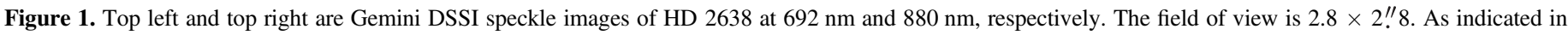

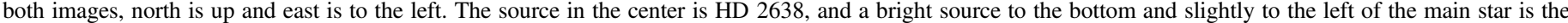

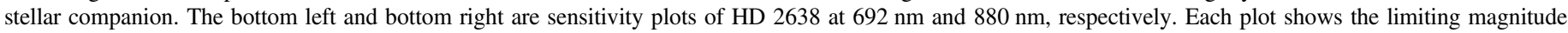

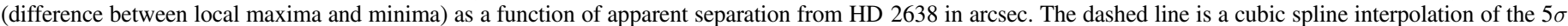
detection limit. Both plots were generated from the corresponding images at the top left and right.

companion to HD 164509, the uncertainty will lie at the upper end of that range. Thus, for separation, we assigned the conservative uncertainty of 2 mas, as shown in Table 2. Similarly, for the position angle, an uncertainty of $\sim 0^{\circ} .2$ is consistent with previous measurements acquired using the Gemini/DSSI configuration. For uncertainties in the magnitude difference between the primary and secondary, we used the empirically determined precision for such measurements provided by Horch et al. (2004).

The sensitivity plots provided in Figures 1 and 2 show the magnitude difference between local maxima and minima in the corresponding image as a function of the separation from the primary host star. The construction of these sensitivity plots are described in more detail by Howell et al. (2011). As shown in the sensitivity plots, the limiting resolution of DSSI with Gemini is $\sim 0$ " 05 . Each of the two stars discussed here, and their corresponding results are described separately below.

\section{1. $H D 2638$}

Prior to submitting this work, we learned that Riddle et al. (2015) have detected HD 2638's stellar companion. We present our results as an independent detection of this companion. Both DSSI images from Figure 1 show a bright source to the bottom and slightly to the left of HD 2638. Based on the magnitude differences from Table 2, the stellar companion appears to be brighter at $880 \mathrm{~nm}$ than it is at $692 \mathrm{~nm}$, implying that the stellar companion is a late-type star. According to Roberts et al. (2015), the companion's spectral type is $\mathrm{M} 1 \mathrm{~V}$, which seems to be in agreement with our assessment. Our calculations of the projected separation between HD 2638 and its companion star yield $25.5 \pm 1.9$ au at $692 \mathrm{~nm}$ and $25.6 \pm 1.9 \mathrm{au}$ at $880 \mathrm{~nm}$, which is close to Roberts et al.'s (2015) 28.5 au physical separation. Note that the apparent close companion to the north of the primary in each image is within the limting resolution of the instrument and is thus an artifact of the speckle image processing. 

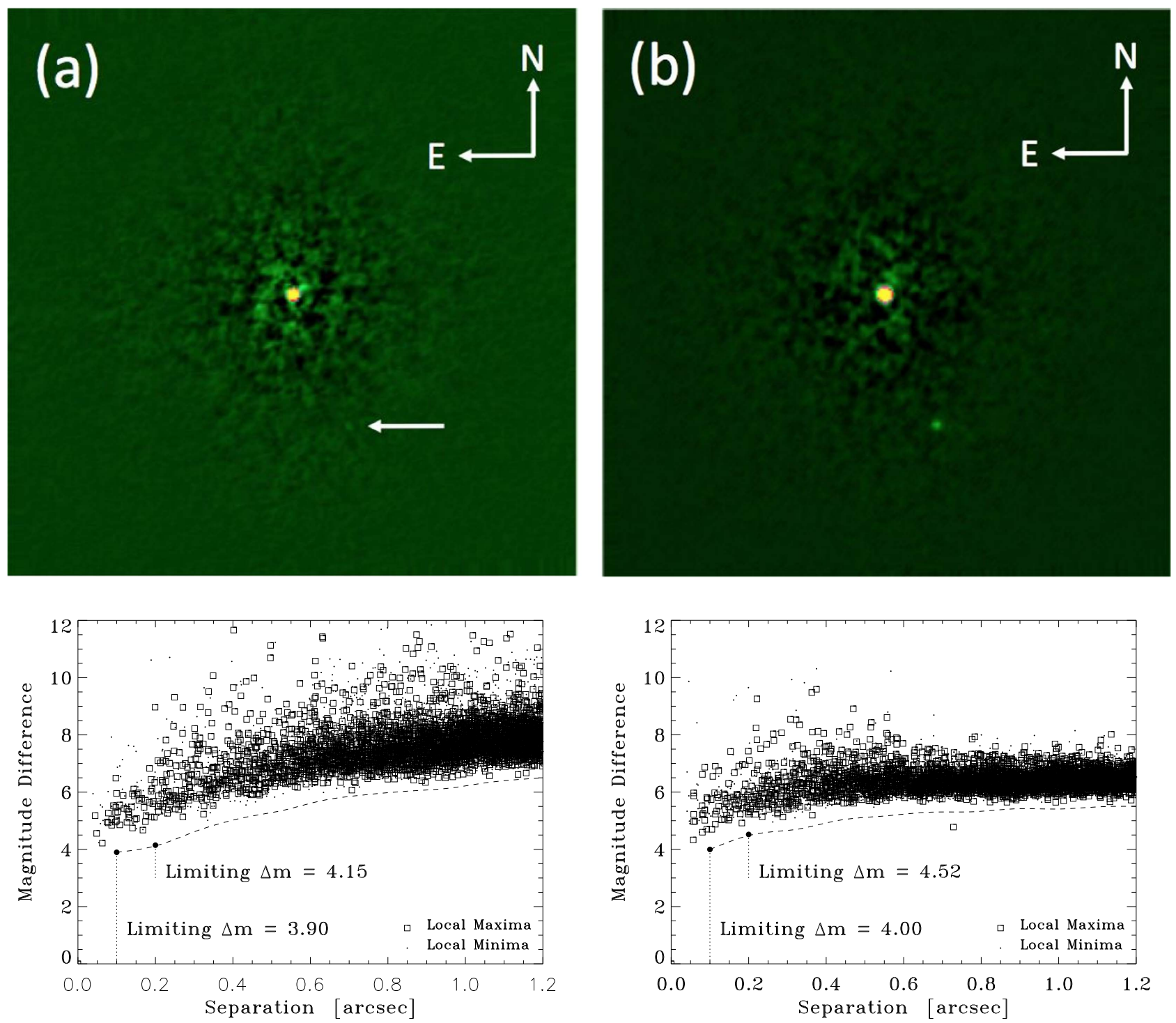

Figure 2. Top left and top right are Gemini DSSI speckle images of HD 164509 at $692 \mathrm{~nm}$ and $880 \mathrm{~nm}$. The field of view is $2.8 \times 2$." 8 . As indicated in both images, north is up and east is to the left. The source in the center is HD 164509, and a bright source to the bottom and right of the main star is the stellar companion. The arrow in the left image indicates the location of the companion. The bottom left and bottom right are sensitivity plots of HD 164509 at $692 \mathrm{~nm}$ and $880 \mathrm{~nm}$, respectively. Each plot shows the limiting magnitude (difference between local maxima and minima) as a function of apparent separation from HD 164509 in arcsec. The dashed line is a cubic spline interpolation of the $5 \sigma$ detection limit. Both plots are generated from the corresponding images at the top left and right.

Table 2

DSSI Astrometry and Photometry Results

\begin{tabular}{|c|c|c|c|c|}
\hline \multirow{2}{*}{ Measurements } & \multicolumn{2}{|c|}{ HD 2638} & \multicolumn{2}{|c|}{ HD 164509} \\
\hline & $692 \mathrm{~nm}$ & $880 \mathrm{~nm}$ & $692 \mathrm{~nm}$ & $880 \mathrm{~nm}$ \\
\hline Position Angle E of $\mathrm{N}\left({ }^{\circ}\right)$ & $167.7 \pm 0.2$ & $167.7 \pm 0.2$ & $202.5 \pm 0.2$ & $202.6 \pm 0.2$ \\
\hline Projected Separation (au) & $25.5 \pm 1.9$ & $25.6 \pm 1.9$ & $36.5 \pm 1.9$ & $36.5 \pm 1.9$ \\
\hline$\Delta m^{\mathrm{a}}$ & $3.83 \pm 0.2$ & $2.80 \pm 0.2$ & $5.53 \pm 0.4$ & $4.41 \pm 0.4$ \\
\hline
\end{tabular}

Note.

a $\Delta m$ is the apparent magnitude difference between the primary and secondary stars.

\subsection{HD 164509}

Figure 2 contains two images that display a source southwest of HD 164509. The magnitude differences of this system imply that the stellar companion is considerably fainter than the host star by a factor of almost 100. In fact, it is so faint at $692 \mathrm{~nm}$ that it is difficult to resolve in the image. Despite the fact that HD 164509 is more luminous than HD 2638, the considerable faintness of HD 164509's companion as compared to HD 2638's implies that this stellar companion is a very cool, late-type star. Based on the data from Table 2, the physical separation between HD 164509 and its companion is 
Table 3

Stellar Companion Isochrone Fitting Results

\begin{tabular}{lcc}
\hline \hline Parameters & HD 2638 & HD 164509 \\
\hline$M_{\star}\left(M_{\odot}\right)$ & $0.48 \pm 0.03$ & $0.42 \pm 0.03$ \\
$R_{\star}\left(R_{\odot}\right)$ & $0.46 \pm 0.02$ & $0.40 \pm 0.02$ \\
$L_{\star}\left(L_{\odot}\right)$ & $0.030 \pm 0.005$ & $0.020 \pm 0.003$ \\
$T_{e}(K)$ & $3571 \pm 48$ & $3446 \pm 43$ \\
$\log g\left(\mathrm{~cm} \mathrm{~s}^{-2}\right)$ & $4.80 \pm 0.02$ & $4.85 \pm 0.02$ \\
\hline
\end{tabular}

$36.5 \pm 1.9 \mathrm{au}$. To compare, the planet Neptune is about $30 \mathrm{au}$ from our Sun, and the result falls short of the dwarf planet Pluto's average distance of $39.5 \mathrm{au}$. Since HD 164509 is slightly more massive than our Sun and with the given distance between the host star and its companion, this leads to credence that the faint object may be gravitationally bound to HD 164509. One interesting thing to point out is that this dim star may be "an additional longer period companion" that Giguere et al. (2012) speculated when they came across the RV data's residual linear trend. As of this writing, there has been no confirmation of HD 164509 hosting a stellar companion.

\subsection{Stellar Isochrone Fitting}

To determine the properties of the detected stellar companions, we performed a stellar isochrone fit using the methodology described by Everett et al. (2015) and Teske et al. (2015). Briefly, the method maps out the probability distribution of the primary star using Dartmouth stellar isochrones. The inputs for this analysis are the stellar properties shown in Table 1. The combination of the resulting probability distributions for the primary with the multi-band observations described in Section 3 produce a probability distribution for the properties of the secondary. Such a result assumes that it is a bound companion that falls on the same isochrone as the primary.

The results of our isochrone fits for the stellar companions are shown in Table 3. The derived stellar properties are consistent with both of the companion stars being late-type

\section{HD 2638}

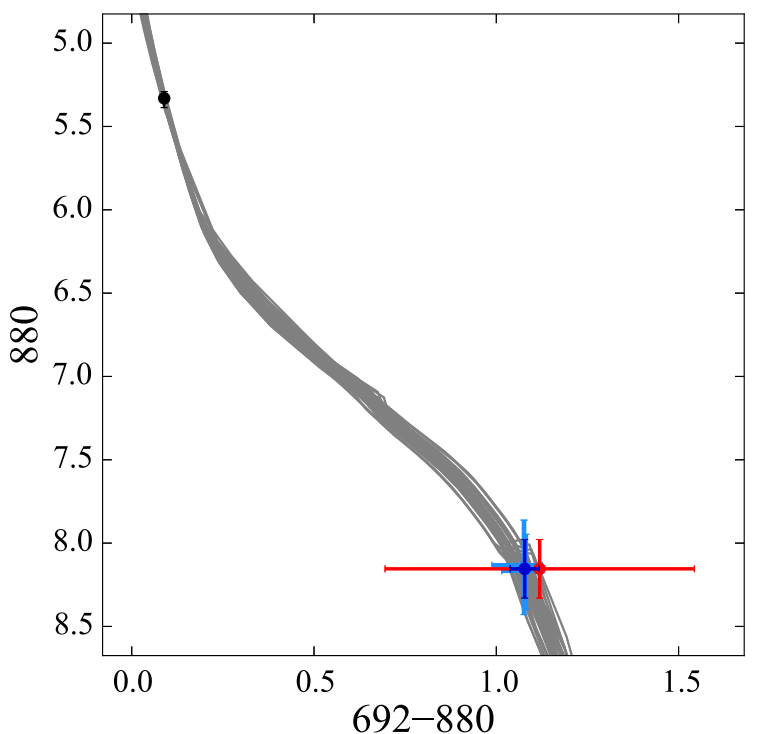

main sequence stars ( $\mathrm{M}$ dwarfs). Note that both our mass determination (Table 3) and projected separation (Table 2) for the HD 2638 stellar companion match well with the results obtained by Roberts et al. (2015). The results from our isochrone fitting are shown in Figure 3 for HD 2638 (left) and HD 164509 (right). The color-magnitude diagrams include the set of isochrones that are within $\pm 1 \sigma$ of the primary star metallicity. The black data point represents the primary star and the red data point shows the location of the secondary based on the measurements described in Section 3. The dark blue data point is the average location of the secondary based on the probability distributions of the isochrone fitting. The location of the secondary from measurements and from isochrone fits is consistent with one another, indicating that the assumption of the secondary being bound to the primary is indeed a valid assumption.

\subsection{Proper Motion and Astrometry}

A further test that the detected companions are indeed bound to the primary is to analyze the common proper motion of the stars on the sky. For HD 2638, such data are available from the "Fourth Interferometric Catalog of Binary Stars" (see description in Hartkopf et al. 2001). The observations of HD 2638 from the catalog span a time frame from 2012.67 to 2015.74. Figure 4 shows the locations of the primary star and secondary star relative to a zero position for the primary at the first epoch shown, 2012.67. The primary star positions are shown as filled circles, and the secondary positions as open squares. The two measurements presented in this work, which on this scale are indistinguishable, are shown in red. Dotted lines link the primary and secondary for the first and last observations in the sequence, and for our $692 \mathrm{~nm}$ observations. The proper motions (see Table 1) are drawn from van Leeuwen (2007). This figure demonstrates that the pair of stars are clearly moving together.

For HD 164509, we have only the single measurement described in this work for the relative astrometry as of now, so the same analysis cannot be completed. However, it is worth

\section{HD 164509}

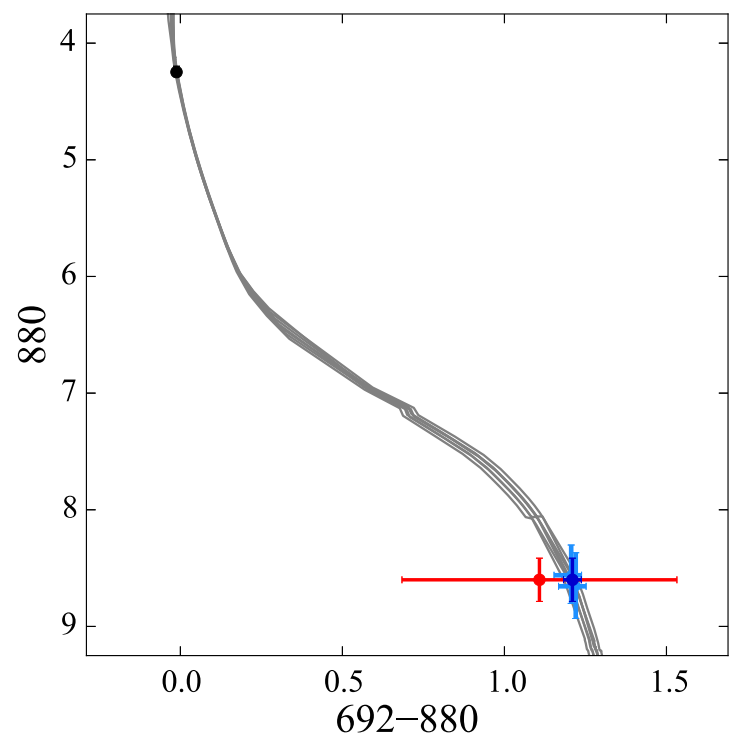

Figure 3. Stellar isochrone models of HD 2638 (left) and HD 164509 (right). The black dot near the top is the primary star, the dark blue dot near the bottom is the average model of the companion, and the red dot is the observed companion. Note that in both cases, the model fits well with the observation. 


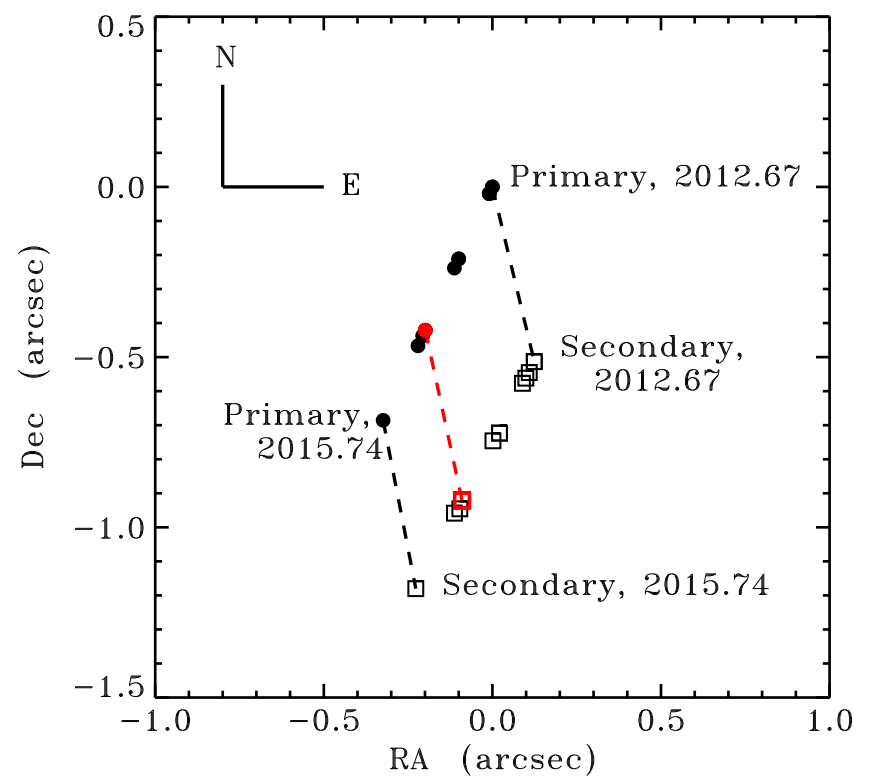

Figure 4. Proper motion of the HD 2638 primary (solid circles) and secondary (open squares) over time. The measurements presented in this work are shown in red. The dashed lines link the primary and secondary for the first and last observations in the sequence, and for our observation.

noting that the proper motion of HD 164509 is significantly smaller than for HD 2638 (see Table 1). Thus, a few more speckle observations of this star over the next few years would allow the same analysis to be undertaken since these numbers, although not as big as for HD 2638, are several times the typical precision for the speckle observations.

\section{IMPLICATIONS FOR THE KNOWN PLANETS}

The presence of stellar companions can pose a significant challenge for orbital stability and formation scenarios for planets in such systems (Ngo et al. 2015; Wang et al. 2015). Issues regarding planet formation include protoplanetary disk truncation, grain condensation, and planetesimal accumulation (see Thebault \& Haghighipour 2014 and references therein). To test whether our observations are consistent with the presence of the planets, we use the orbital dynamics results of Holman \& Wiegert (1999) for test particles in binary systems. Specifically,

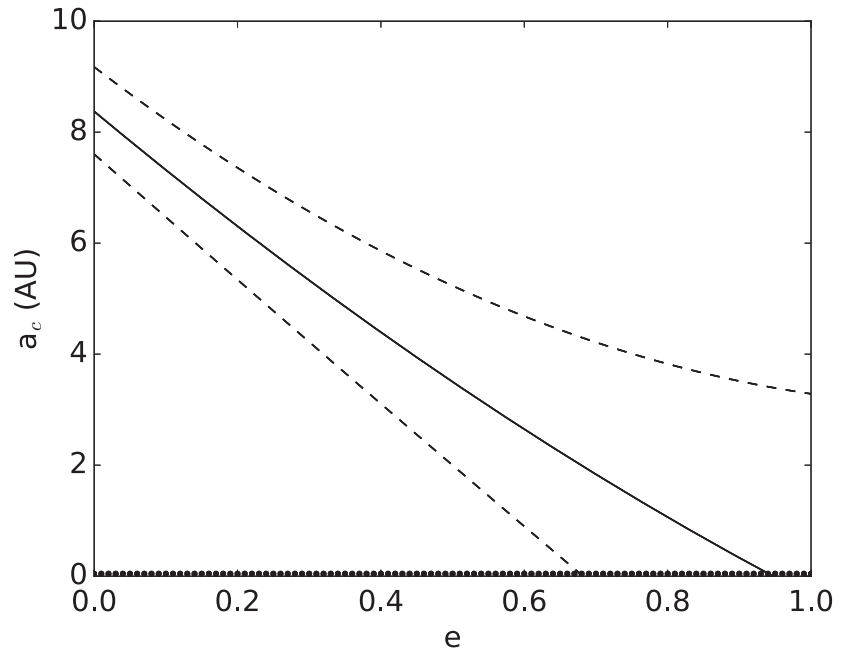

we calculate the critical semimajor axis, $a_{c}$, beyond which planetary orbits would be unstable in the systems. The resulting plots of $a_{c}$ as a function of binary eccentricity $e$ are shown in Figure 5. The values of $a_{c}$ were calculated using Equation (1) from Holman \& Wiegert (1999):

$$
\begin{aligned}
a_{c}= & {[(0.464 \pm 0.006)+(-0.380 \pm 0.010) \mu} \\
& +(-0.631 \pm 0.034) e+(0.586 \pm 0.061) \mu e \\
& +(0.150 \pm 0.041) e^{2} \\
& \left.+(-0.198 \pm 0.074) \mu e^{2}\right] a_{b}
\end{aligned}
$$

where $a_{b}$ is the binary semimajor axis. The mass ratio, $\mu$, is calculated as $\mu=m_{2} /\left(m_{1}+m_{2}\right)$, where $m_{1}$ and $m_{2}$ are the masses of the primary and secondary, respectively. Using the values from Tables 2 and 3, we have $\mu=0.357 \pm 0.009$ and $a_{b}=25.5 \mathrm{au}$ for HD 2638, and $\mu=0.274 \pm 0.006$ and $a_{b}=36.5 \mathrm{au}$ for HD 164509. Note that this assumes the projected separations are the true semimajor axes of the binary companions. Including both the uncertainties in Equation (1) and $\mu$, we include lines for the $1 \sigma$ uncertainties as dashed lines in Figure 5. The semimajor axes of the known planets (see Table 1) are represented in each case by a horizontal dotted line. These figures show that the stability of the planetary orbits remain secure for most values of the binary eccentricity. The maximum binary eccentricities (where the planetary semimajor axis lines intersect the eccentricity lines) are $e=0.94 \pm 0.26$ and $e=0.87 \pm 0.21$ for HD 2638 and HD 164509, respectively.

Given that the planets were discovered with the RV technique, it is worth pausing to consider the effect of the stellar binary companions on the planetary interpretation of the RV data. Using the stellar parameters of the primary and secondary from Tables 1 and 3 respectively, along with the projected separations from Table 2, we calculate the expected orbital periods and RV semi-amplitudes for each system. For HD 2638, the minimum orbital period is $\sim 110$ years with a maximum RV semi-amplitude of $\sim 2.4 \mathrm{~km} \mathrm{~s}^{-1}$. For HD 164509, the minimum orbital period is $\sim 180$ years with a maximum RV semi-amplitude of $\sim 1.7 \mathrm{~km} \mathrm{~s}^{-1}$. As noted in Section 2, the Kepler solution to the HD 164509 RVs includes a linear trend, although the time baseline since discovery is

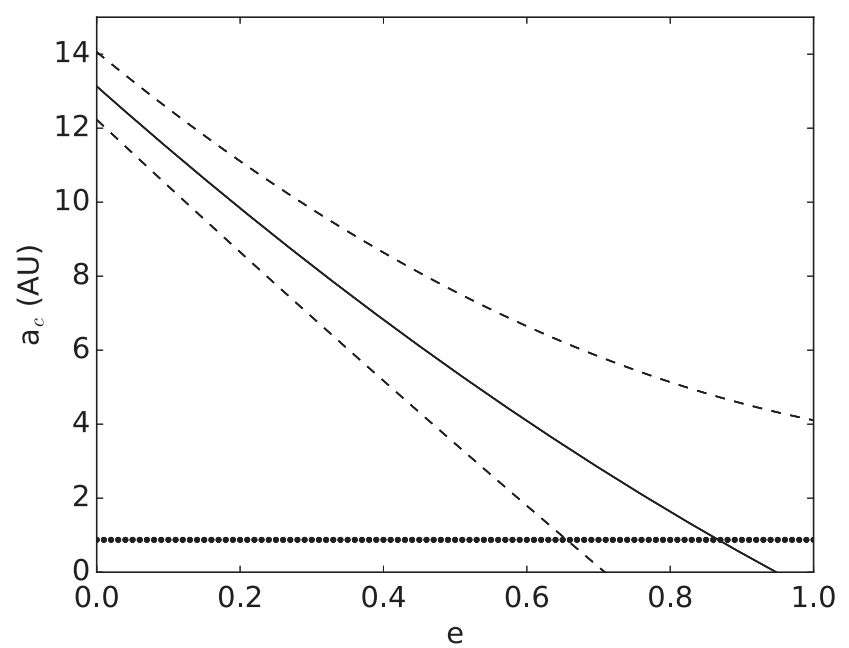

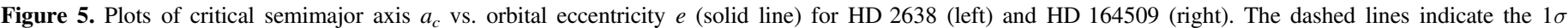
uncertainties in the relationship and the horizontal dotted lines represent the semimajor axes of the known planets. 
insufficient to charactize the nature of the trend. Assuming the minimum separations above, the companions cannot be confused with the planetary signals and thus have no effect on the planetary interpretation of the RV data.

\section{CONCLUSION}

Determining the stellar architecture of planetary systems is an ongoing process, improving as the capability to detect faint stellar companions increases. Stellar binarity can have a profound effect on exoplanetary systems, both in terms of formation processes and long-term orbital stability. Thus, determining the binarity of known exoplanet host stars is a critical step in the characterization of those systems.

Here we have presented detections of stellar companions to two known exoplanet host stars: HD 2638 and HD 164509. Though the stellar companion to HD 2638 was previously detected by Roberts et al. (2015), the new data from DSSI will provide additional information of the astrometry of the companion and the stellar properties, given that the passbands used are particular to the DSSI camera. We have shown that the detected companions have properties consistent with them both being $\mathrm{M}$ dwarfs, and the isochrone analysis shows that they are both likely to be gravitationally bound to the host stars. Fortunately, the presence of the stellar companions do not pose serious orbital stability problems for the known exoplanets, making the overall architecture of the systems self-consistent. These planetary systems represent additional interesting examples of planet formation and evolution in the presence of multiple stars.

Based on observations obtained at the Gemini Observatory, which is operated by the Association of Universities for Research in Astronomy, Inc., under a cooperative agreement with the NSF on behalf of the Gemini partnership: the National Science Foundation (United States), the National Research Council (Canada), CONICYT (Chile), the Australian Research Council (Australia), Ministério da Ciência, Tecnologia e Inovação (Brazil) and Ministerio de Ciencia, Tecnología e Innovación Productiva (Argentina). This research has made use of the NASA Exoplanet Archive, which is operated by the California Institute of Technology, under contract with the National Aeronautics and Space Administration under the Exoplanet Exploration Program. The results reported herein benefited from collaborations and/or information exchange within NASA's Nexus for Exoplanet System Science (NExSS) research coordination network sponsored by NASA's Science Mission Directorate.

\section{REFERENCES}

Benest, D. 1988, A\&A, 206, 143

Benest, D. 1989, A\&A, 223, 361

Benest, D. 1996, A\&A, 314, 983

Bonfanti, A., Ortolani, S., \& Nascimbeni, V. 2016, A\&A, 585, A5

Cartier, K. M. S., Gilliland, R. L., Wright, J. T., \& Ciardi, D. R. 2015, ApJ, 804, 97

Ciardi, D. R., Beichman, C. A., Horch, E. P., \& Howell, S. B. 2015, ApJ, 805,16

Eggenberger, A., Udry, S., \& Mayor, M. 2004, A\&A, 417, 353

ESA 1997, The Hipparcos and Tycho Catalogues (Noordwijk: ESA) ESASP 1200

Everett, M. E., Barclay, T., Ciardi, D. R., et al. 2015, AJ, 149, 55

Giguere, M. J., Fischer, D. A., Howard, A. W., et al. 2012, ApJ, 744, 4

Gilliland, R. L., Cartier, K. M. S., Adams, E. R., et al. 2015, AJ, 149, 24

Ginski, C., Mugrauer, M., Seeliger, M., et al. 2016, MNRAS, 457, 2173

Hartkopf, W. I., McAlister, H. A., \& Mason, B. D. 2001, AJ, 122, 3480

Holman, M. J., \& Wiegert, P. A. 1999, AJ, 117, 621

Horch, E. P., Gomez, S. C., Sherry, W. H., et al. 2011, AJ, 141, 45

Horch, E. P., Howell, S. B., Everett, M. E., Ciardi, D. R., et al. 2012, AJ, 144,165

Horch, E. P., Meyer, R. D., \& van Altena, W. F. 2004, AJ, 127, 1727

Horch, E. P., van Altena, W. F., Demarque, P., et al. 2015, AJ, 149, 151

Horch, E. P., Veillette, D. R., Baena, G., et al. 2009, AJ, 137, 5057

Howell, S. B., Everett, M. E., Sherry, W., Horch, E., \& Ciardi, D. R. 2011, AJ, 142,19

Kane, S. R., Barclay, T., Hartmann, M., et al. 2015, ApJ, 815, 32

Kraus, A. L., Ireland, M. J., Huber, D., Mann, A. W., \& Dupuy, T. J. 2016, AJ, 152,8

Lohmann, A. W., Weigelt, G., \& Wirnitzer, B. 1983, ApOpt, 22, 4028

Meng, J., Aitken, G. J. M., Hege, E. K., \& Morgan, J. S. 1990, JOSAA, 7, 1243

Moutou, C., Mayor, M., Bouchy, F., et al. 2005, A\&A, 439, 367

Ngo, H., Knutson, H. A., Hinkley, S., et al. 2015, ApJ, 800, 138

Raghavan, D., McAlister, H. A., Henry, T. J., et al. 2010, ApJS, 190, 1

Riddle, R. L., Tokovinin, A., Mason, B. D., et al. 2015, ApJ, 799, 4

Roberts, L. C., Tokovinin, A., Mason, B. D., et al. 2015, AJ, 149, 118

Shaya, E. J., \& Olling, R. P. 2011, ApJ, 192, 2

Sirothia, S. K., Lecavelier des Etangs, A., Gopal-Krishna, Kantharia, N. G., \& Ishwar-Chandra, C. H. 2014, A\&A, 562, A108

Teske, J. K., Everett, M. E., Hirsch, L., et al. 2015, AJ, 150, 144

Thebault, P., \& Haghighipour, N. 2014, in Planetary Exploration and Science: Recent Advances and Applications, ed. S. Jin, N. Haghighipour, \& W.-H. Ip (Berlin: Springer) arXiv:1406.1357

van Leeuwen, F. 2007, A\&A, 474, 653

Wang, J., Fischer, D. A., Horch, E. P., \& Xie, J.-W. 2015, ApJ, 806, 248

Wang, J., \& Ford, E. B. 2011, MNRAS, 418, 1822

Zucker, S., \& Mazeh, T. 2002, ApJL, 568, L113 